\title{
On the self-consistent physical parameters of LMC intermediate-age clusters
}

\author{
Leandro O. Kerber ${ }^{1,2}$ and Basílio X. Santiago ${ }^{3}$ \\ ${ }^{1}$ IAG/USP, São Paulo, Brazil \\ email: kerber@astro.iag.usp.br \\ ${ }^{2}$ INAF-OAPd, Padova, Italy \\ ${ }^{3}$ IF/UFRGS, Porto Alegre, Brazil
}

\begin{abstract}
The LMC clusters with similar ages to the Milky Way open clusters are in general more metal-poor and more populous than the latter, being located close enough to allow their stellar content to be well resolved. Therefore, they are unique templates of simple stellar population (SSP), being crucial to calibrate models describing the integral light as well as to test the stellar evolution theory. With this in mind we analyzed $H S T / \mathrm{WFPC} 2(V, B-V)$ colour-magnitude diagrams (CMDs) of 15 populous LMC clusters with ages between $\sim 0.3$ Gyr and $\sim 4$ Gyr using different stellar evolutionary models. Following the approach described by Kerber, Santiago \& Brocato (2007), we determined accurate and self-consistent physical parameters (age, metallicity, distance modulus and reddening) for each cluster by comparing the observed CMDs with synthetic ones generated using isochrones from the PEL and BaSTI libraries. These determinations were made by means of simultaneous statistical comparison of the main-sequence fiducial line and the red clump position, offering objective and robust criteria to select the best models. We compared these results with the ones obtained by Kerber, Santiago \& Brocato (2007) using the Padova isochrones. This revealed that there are significant trends in the physical parameters due to the choice of stellar evolutionary model and treatment of convective core overshooting. In general, models that incorporate overshooting presented more reliable results than those that do not. Furthermore, the Padova models fitted better the data than the PEL and BaSTI models. Comparisons with the results found in the literature demonstrated that our derived metallicities are in good agreement with the ones from the spectroscopy of red giants. We also confirmed that, independent of the adopted stellar evolutionary library, the recovered 3D distribution for these clusters is consistent with a thick disk roughly aligned with the LMC disk as defined by field stars. Finally, we also provide new estimates of distance modulus to the LMC center, that are marginally consistent with the canonical value of $18.50 \mathrm{mag}$.
\end{abstract}

Keywords. Hertzsprung-Russell diagram, galaxies: individual (LMC), Magellanic Clouds, galaxies: star clusters

\section{Introduction}

The LMC contains a rich system of stellar clusters, with more than three thousand cataloged objects (Bica et al. 2008) and covering ages from few Myr to about 13 Gyr. There are about one hundred that can be considered as populous ones $\left(>10^{5}\right.$ stars), which offer the opportunity to recover the age-metallicity relation for the LMC by means of accurate age (from CMD analysis) and metallicity (from spectroscopy analysis) determinations. Furthermore, the objects with ages between $\sim 0.3$ and $4 \mathrm{Gyr}$ - the intermediate-age LMC clusters (IACs) - are more metal poor than the open clusters in the Milky Way, being therefore fundamental pieces in the local universe to calibrate integrated light models as well as to test the evolutionary models in the sub-solar metallicity regime. 


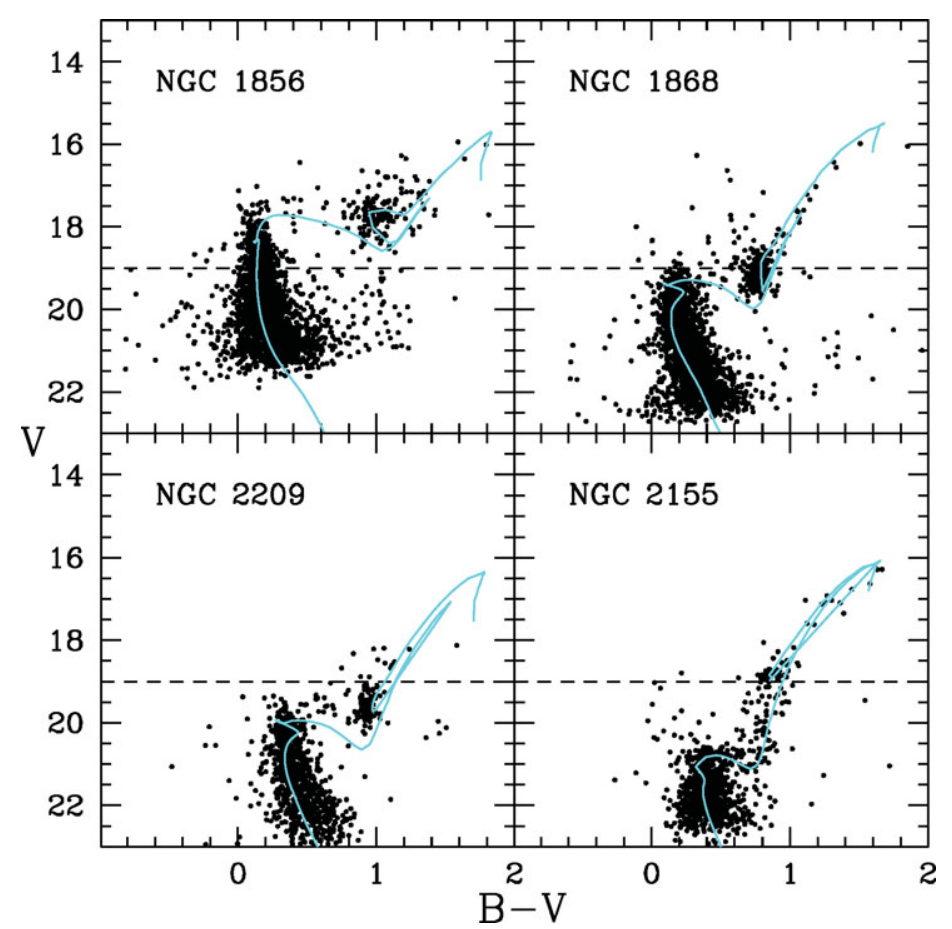

Figure 1. Examples of observed $H S T / W F P C 2(V, B-V)$ CMDs for 4 stellar clusters in our sample. These clusters are in a sequence of age, from $\sim 0.3$ Gyr (NGC 1856) to $\sim 3.5$ Gyr (NGC 2155). The best solutions found by us using the Padova isochrones (KSB07) are also shown in the figure.

Taking the advantage of the superior photometric quality of the HST and the intrinsic large stellar statistics for the populous IACs we have applied a statistical method to recover accurate physical parameters - not only age, but also metallicity, distance and reddening - for these objects in a self-consistent way. By self-consistency we mean the ability to simultaneously infer these parameters from the same data-set without any prior assumptions about any of them. The method, presented by Kerber, Santiago \& Brocato (2007) (hereafter KSB07) using the Padova isochrones (Girardi et al. 2002), joins CMD modelling and statistical analysis to objectively determine which are the synthetic CMDs that best reproduce the observed ones. In the present work we expanded this analysis to two other stellar evolutionary libraries: PEL (or Pisa, Castellani et al. 2003) and BaSTI (or Teramo, Pietrinferni et al. 2004). This allowed us to quantify how the recovered physical parameters depend on the adoption of different stellar evolutionary libraries, including the treatment for the convective core overshooting. Furthermore, since we are determining the individual distance to each cluster, we could also probe the three dimensional distribution of these clusters, which seems to be roughly aligned with LMC disk (KSB07; Grocholski et al. 2007), and to obtain new determinations of distance modulus to the LMC centre.

\section{CMDs: data vs. model}

$H S T / W F P C 2$ data. We analysed HST/WFPC2 $(V, B-V)$ CMDs for a sample of 15 IACs in the LMC covering ages from $\sim 0.3$ to $\sim 4$ Gyr. These data come from Brocato et al. (2001) and are photometrically quite homogeneous, reaching typically $V \sim 22$ mag. 
Table 1. The adopted stellar evolutionary libraries.

\begin{tabular}{lccc}
\hline & Padova & PEL & BaSTI \\
\hline & & $($ Pisa $)$ & (Teramo) \\
\hline Convective core: & & & \\
Classical $\left(\Lambda_{\mathrm{OV}} / H_{\mathrm{p}}=0\right) ?$ & no & yes & yes \\
Overshooting $\Lambda_{\mathrm{OV}} / H_{\mathrm{p}} \neq 0$ & $\sim 0.25$ & 0.25 & 0.25 \\
\hline$Z\left(0.001-Z_{\odot}\right)$ & $0.001,0.002,0.004,0.006$ & $0.001,0.004$ & $0.001,0.002,0.004$ \\
& $0.008,0.012,0.015,0.019$ & 0.008 & $0.008,0.010,0.0198$ \\
& $(8$ values $)$ & $(3$ values $)$ & $(6$ values $)$ \\
\hline Age $(0.10-4 \mathrm{Gyr})$ & 33 values & 24 values & 22 values \\
\hline
\end{tabular}

As can be seen in Fig. 1, these CMDs cover at least 2 magnitudes along the main sequence (MS) and clearly display the core helium burning stars in the red clump (RC).

CMD modelling. We are modelling these CMDs as SSPs, where the basic steps to generate a synthetic CMD are the following: 1) we choose the stellar evolutionary model; 2) we choose an age and metallicity. These two steps are equivalent to picking up an isochrone from the adopted stellar evolutionary library; 3) we apply our choice of distance modulus and reddening to the isochrone; 4) we distribute the synthetic stars following a Salpeter IMF and a fraction of binaries of $30 \%$; 5 ) as a last step we introduce the photometric errors and completeness as determined from the data.

The stellar evolutionary libraries. In Table 1 we can see the basic differences in the three stellar evolutionary libraries that were used. All of them offer models with treatment for convective core overshooting with similar values for $\Lambda_{\mathrm{OV}}$. However, only PEL and BaSTI also offer models without overshooting - the classical or canonical models. Another important difference between the libraries is their discreteness or the number of steps in age or metallicity. In this respect Padova offers a significant larger number of possibilities than the other libraries, specially PEL, where there are only three values of metallicity available.

Statistical comparisons. To determine the best models we applied a statistical tool to compare simultaneously the observed and synthetic MS and RC. For the MS we compared the differences in colour along the observed and theoretical fiducial lines of each cluster using a $\chi^{2}$ statistics to assess their similarity. For the evolved stars we computed a CMD distance, $\delta_{\mathrm{RC}}$, between the median positions of real and artificial RC stars. So, the best models are the ones that have the minimum values in both statistics.

\section{Self-consistent physical parameters}

Table 2 summarises the results. The first line lists the typical formal random uncertainties in each parameter. For more details on how these uncertainties are estimated we refer to KSB07. In the remaining lines, the first (second) number in each entry is the systematic (rms) difference in the specific comparison being made. We compared our previous results based on Padova with the literature, as well as the distinct evolutionary models among themselves. We detail these comparisons below.

Padova vs. literature. Figure 2 shows the comparisons between our results using the Padova isochrones (KSB07) with the ones found in the literature. We identify an underestimation in age in the previous works, which were based on ground-based observations (e.g., Elson \& Fall 1988). The amplitude of the effect is $\sim 0.30$ in $\log$ (age) for clusters 


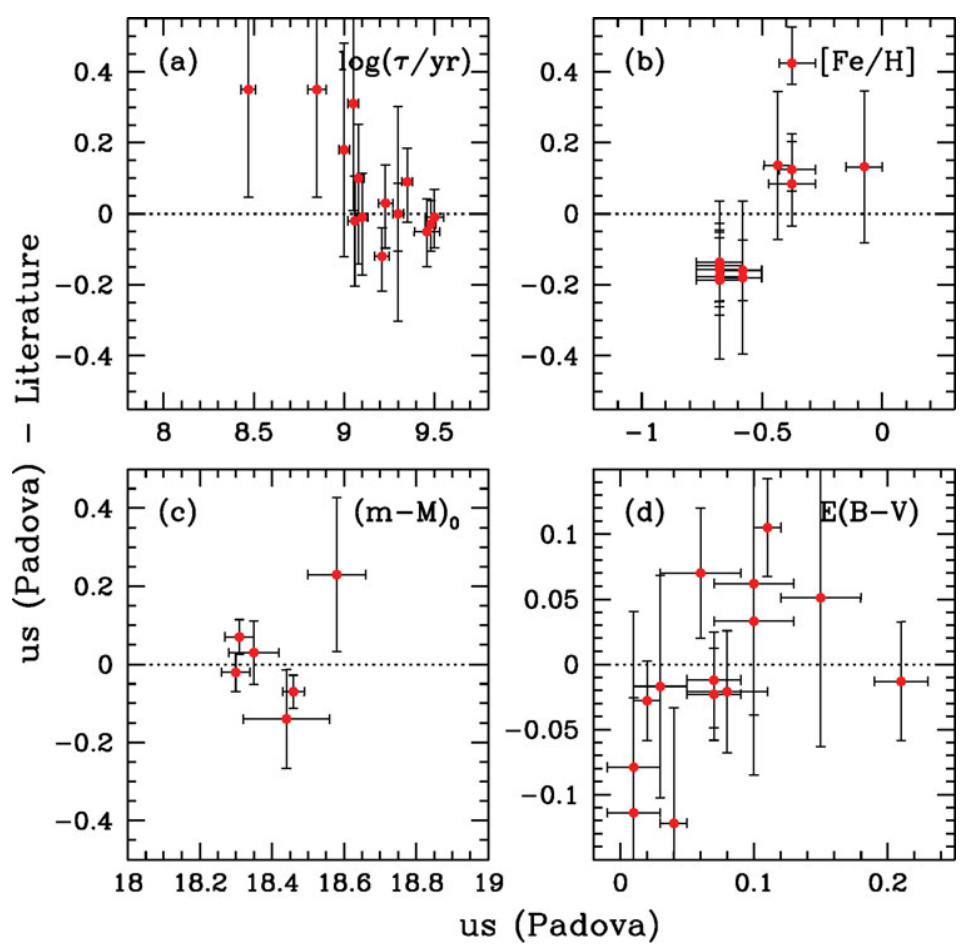

Figure 2. Differences in the physical parameters determined by us (using the Padova isochrones) in relation to the ones found in the literature (see text for details).

younger than 1 Gyr. On the other hand, for the clusters older than this age limit, all of them analysed with high quality data $(H S T$ or $V L T)$ by other authors (e.g., Rich et al. 2001), this difference seems disappear.

A very interesting comparison can be done based on spectroscopy of red giants, in particular using the analysis of Calcium triplet (Olszewski et al. 1991; Grocholski et al. 2006). These comparisons reveal that, despite the scatter of 0.20 , our CMD analysis predicts metallicities without any systematic effect. So, this scatter can be considered as a realistic random uncertainty in our method based on a pure CMD analysis.

Concerning the distance modulus, another interesting comparison can be done with the results based on the $\mathrm{RC}$ in the $\mathrm{K}$ band as a distance indicator (Grocholski et al. 2007). For the six stellar clusters in common, despite the significant scatter found, the systematic differences are very small. The same can be said in relation to the reddening, where in this case the literature results come from analysis of integrated optical colours (McLaughlin \& van der Marel 2005).

Classical vs. overshooting. As expected (Gallart, Zoccali \& Aparicio 2005), inside the same library, models with treatment for the convective core overshooting recovered older ages in relation to the ones obtained by the classical models. Despite the systematic differences in $\log$ (age) of 0.08 for the PEL models and 0.13 for the BaSTI models, there is no clear trend in the other parameters.

PEL or BaSTI vs. Padova. The comparisons involving different libraries with convective core overshooting reveal that the adoption of a specific stellar evolutionary model can produce a significant bias in the physical parameters. Taking the Padova library as a reference, the PEL library systematically overestimates distance modulus by 0.12 (or $\sim 2.8 \mathrm{kpc}$ at $50 \mathrm{kpc}$ ), whereas the BaSTI models systematically overestimate ages by 
Table 2. Self-consistent physical parameters. First line: typical formal uncertainties. Other lines: systematic and rms differences between the results from different stellar evolutionary models.

\begin{tabular}{lcccc}
\hline & $\log ($ age $/ \mathrm{yr})$ & {$[\mathrm{Fe} / \mathrm{H}]$} & $(m-M)_{0}$ & $E(B-V)$ \\
\hline typical formal uncertainties & 0.05 & 0.10 & 0.08 & 0.02 \\
\hline Padova - literature & $+0.30 / 0.10^{1}$ & $+0.02 / 0.20$ & $-0.02 / 0.13$ & $-0.01 / 0.07$ \\
& $+0.02 / 0.11^{2}$ & & & \\
\hline
\end{tabular}

\begin{tabular}{llllll}
\hline $\begin{array}{l}\text { PEL } \\
\text { overshooting - classical }\end{array}$ & $+0.08 / 0.09$ & $+0.06 / 0.11$ & $+0.01 / 0.10$ & $-0.02 / 0.03$ \\
\hline $\begin{array}{l}\text { BaSTI } \\
\text { overshooting - classical }\end{array}$ & $+0.13 / 0.09$ & $+0.02 / 0.20$ & $+0.04 / 0.14$ & $0.00 / 0.04$ \\
\hline PEL (over) - Padova & $+0.00 / 0.06$ & $+0.04 / 0.17$ & $+0.12 / 0.11$ & $0.00 / 0.04$ \\
\hline BaSTI (over) - Padova & $+0.12 / 0.05$ & $+0.06 / 0.12$ & $+0.03 / 0.05$ & $-0.01 / 0.03$ \\
\hline
\end{tabular}

Notes: ${ }^{1}$ age $<1$ Gyr $;{ }^{2}$ age $>1$ Gyr.

0.12 in $\log$ (age) ( $\sim 0.30$ Gyr for an age of 1 Gyr). Apart from these, no other trend was observed in the other parameters.

\section{The quality of the fit}

To determine the quality of the fit we took simultaneously the MS and RC fit into account. We define a parameter called $n$ for each model as

$$
n=\sqrt{\left(\chi^{2} / \sigma_{\chi}\right)^{2}+\left(\delta_{R C} / \sigma_{\delta}\right)^{2}},
$$

where $\sigma$ means the standard deviation in each statistics as determined by control experiments. So, the best model is the one that minimizes this parameter $\left(n_{\min }\right)$.

As can be seen in Fig. 3, there is no clear age dependence in the quality of the fit. On the other hand, on average, models with overshooting fit better the data than the classical ones. Furthermore, the quality of the fit is similar for the PEL and BaSTI libraries, but significant higher for the Padova models.

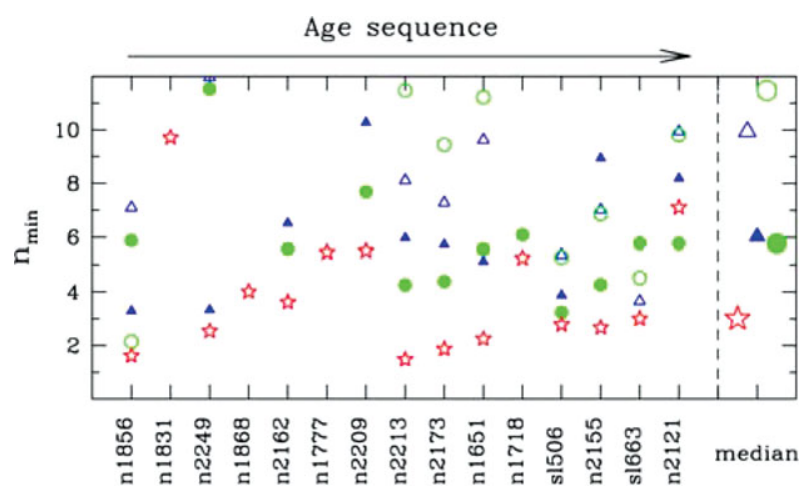

Figure 3. The quality of the fit for all clusters as determined by the $n_{\min }$. The clusters are ordered according to age, with the youngest on the left. The symbols code the different choices of stellar evolutionary models: Padova (stars), PEL with (solid triangles) and without (open triangles) overshooting, BaSTI with (solid circles) and without (open circles) overshooting. The median values for all clusters are also shown in the figure. 


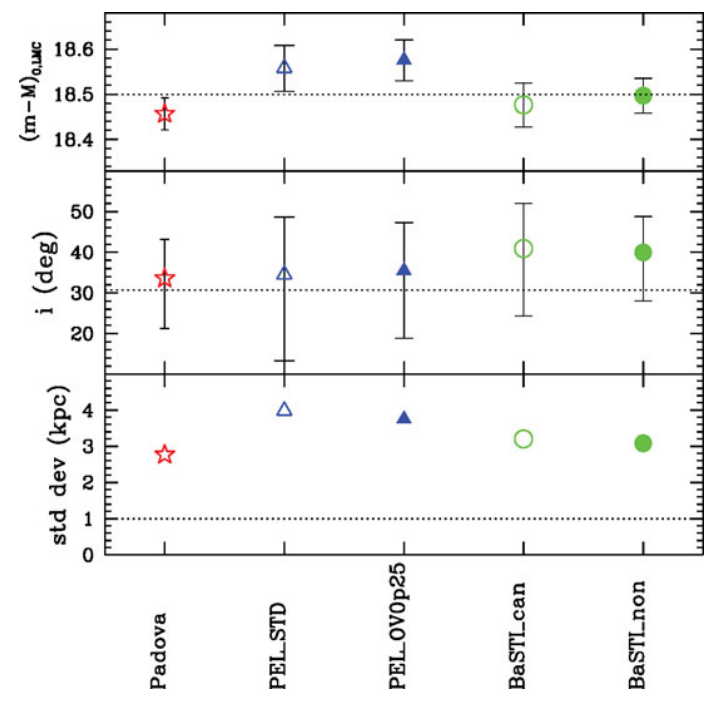

Figure 4. LMC distance (upper panel), disk inclination (middle panel) and standard deviation (lower panel) in the fitted 3D distribution for our sample of 15 stellar clusters.

\section{3D distribution and LMC distance}

Since we determined the individual distances to each cluster we were able to probe the $3 \mathrm{D}$ distribution of these objects and also to provide new determinations of the distance to the LMC centre. These results are summarized in Fig. 4, which reveals that: 1) independent of the adopted stellar evolutionary library, the IACs have a spatial distribution roughly aligned (disk inclination $\sim 35^{\circ}$ ) with the LMC disk as defined by field stars (in this case Cepheid stars, Nikolaev et al. 2004); 2) the IACs seem to belong to a thick disk, and the spatial scatter around the best-fit geometry is slightly smaller for models with overshooting; 3 ) the determined distance moduli to the LMC center are marginally consistent with the canonical value of $18.50 \mathrm{mag}$ (Clementini et al. 2003); the PEL models recovered systematically larger values than the ones obtained using the other libraries.

\section{References}

Bica, E., Bonatto, C., Dutra, C. M., \& Santos, J. F. C. 2008, MNRAS, 389, 678

Brocato, E., Di Carlo, E., \& Menna, G. 2001, A\& $\&$, 374, 523

Castellani, V., Degl'Innocenti, S., Marconi, M., Prada Moroni, P. G., \& Sestito, P. 2003, A\&\&A, 404,465

Clementini, G., Gratton, R., Bragaglia, A., et al. 2003, AJ, 125, 1309

Elson, R. A. \& Fall, S.M. 1988, AJ, 96, 1383

Gallart, C., Zoccali, M., \& Aparicio, A. 2005, ARAA, 43, 387

Girardi, L., Bertelli, G., Bressan, A., et al. 2002, A\&A, 391, 195

Grocholski, A. J., Cole, A. A., Sarajedini, A., Geisler, D., \& Smith, V. V. 2006, AJ, 132, 1630

Grocholski, A. J., Sarajedini, A., Olsen, K., Tiede, G., \& Mancone, C. 2007, AJ, 134, 680

Kerber, L. O., Santiago, B. X., \& Brocato, E. 2007, A\&A, 462, 139 (KSB07)

McLaughlin, D. E., \& van der Marel, R. P. 2005, AJ, 161, 304

Nikolaev, S., Drake, A. J., Keller, S. C., et al. 2004, ApJ, 601, 260

Olszewski, E. W., Schommer, R. A., Suntzeff, B., \& Harris, H. 1991, AJ, 101, 515

Pietrinferni, A., Cassisi, S., Salaris, M., \& Castelli, F. 2004, ApJ, 612, 168

Rich, R. M., Shara, M. M., \& Zurek, D. 2001, AJ, 122, 842 